\title{
Endoscopic Decompression of the Median Nerve for Idiopathic Carpal Tunnel Syndrome
}

\author{
Hamdy Mohammed Behairy \\ Department of Neurosurgery, Al-Azhar University, Cairo, Egypt \\ Email: dr.hamdybehairy@hotmail.com
}

How to cite this paper: Behairy, H.M. (2018) Endoscopic Decompression of the Median Nerve for Idiopathic Carpal Tunnel Syndrome. Neuroscience \& Medicine, 9, $1-8$.

https://doi.org/10.4236/nm.2018.91001

Received: December 18, 2017

Accepted: March 3, 2018

Published: March 6, 2018

Copyright $\odot 2018$ by author and Scientific Research Publishing Inc. This work is licensed under the Creative Commons Attribution International License (CC BY 4.0).

http://creativecommons.org/licenses/by/4.0/

\begin{abstract}
Carpal tunnel syndrome is one of the commonest entrapment neuropathies. Hand pain and numbness are mostly the presenting symptoms. Endoscopic carpal tunnel release (ECTR) has been demonstrated to reduce recovery time and allow faster return to the work. The purpose of this prospective descriptive study was to evaluate the efficacy and advantages of (ECTR) through single proximal incision. In this study, the procedure was done for 36 hands in 36 patients. The results showed that females:male $=9: 1$, mean age was 42 years. Right hand dominance was $90 \%$ and affected in $67 \%$. Preoperatively, the condition was moderate in $61 \%$ and severe in $39 \%$. The average operating time was 10 minutes and the mean follow up period was 6 months with no major postoperative complications. In $8 \%$, transient neurapraxia was found and resolved in 1 week and tenderness was found in $70 \%$ and resolved in 4 weeks. We concluded that endoscopic carpal tunnel release is an effective minimal incisional safe procedure with a high success rate, rapid return to work.
\end{abstract}

\section{Keywords}

Carpal Tunnel Syndrome, Endoscopic Release

\section{Introduction}

Idiopathic carpal tunnel syndrome is the commonest one of the many entrapment neuropathies encountered by neurosurgeons in Egypt and all over the world. It was reported that idiopathic carpal tunnel syndrome accounts for more than 200,000 surgical procedures per year in the United States [1]. Also, it was reported that idiopathic carpal tunnel syndrome affects up to $1 \%$ of the general population and that surgical treatment continues to be the most effective treatment for this condition [2].

Idiopathiccarpal tunnel syndrome was first described as a clinical condition by 
Paget in 1854 and first surgery for tunnel release was performed in 1924 by Herbert Galloway [3]. The most common complications noticed after open procedure were hypertrophic or painful scars and pillar pain (pain in the thenar or hypothenar eminences). Since that, numerous advances have been made to refine this procedure [3]. In 1989, anoriginal endoscopic technique to release the transverse carpal ligament from within the carpal canal attempting to diminish the complications associated with open carpal tunnel release (OCTR) was presented. The early technique of endoscopic carpal tunnel release (ECTR) had a high incidence of complications due to little experience [4]. Later on, a single incision technique that allowed for visualization of the transverse carpal ligament throughout the procedure was developed. With better visualization and increasing surgical experience, the complications associated with ECTR diminished with more rapid return to work and daily activities and less scar tenderness [5] [6]. Different techniques described for carpal tunnel release includes: 1) Open carpal tunnel release. 2) Endoscopic carpal tunnel release via proximal 1 portal (Okutsu, Agee and Menon), Distal 1 portal (Mirza), 2 portals (Chow, Resnick, Brown, Others). 3) Limited incision technique (LIT) 1 incision (Naso, Bromley), 2 incisions (Biyani, Wilson).Proximal 1 portal technique of Men on was the chosen one in this study.

It was described that endoscopic carpal tunnel release with use of palmar portal approach through superficial plane to enable a view from above the transverse carpal ligament aiming to prevent compression of the median nerve inside the already narrow tunnel [7].

Two techniques are the commonly used for ECTR, the two-portal technique of Chow and the single proximal portal technique of Agee. Both of these techniques involve the placement of a blunt instrument beneath the deep transverse carpal ligament and division of it from within the carpal canal, leaving the palmar structures and skin intact with visualization via the endoscope.

Anatomically, the carpal canal is bordered on three sides by bone and on the top by the unyielding, tough, deep transverse carpal ligament. The limits of the transverse carpal ligament correspond to bony attachments to the pisiform proximally and the hook of the hamate distally on the ulnar side and to the tuberosity of the scaphoid and by some fibers to styloid process of radius proximallyand ridge of the trapezium distally on the radial side. The transverse carpal ligament is normally $1-2 \mathrm{~mm}$ in thickness, $2-3 \mathrm{~cm}$ in width and $5 \mathrm{~cm}$ in length. The narrowest portion of the tunnel is at the level of the hook of the hamate. The terms transverse carpal ligament and flexor retinaculum are not synonymous. The flexor retinaculum is composed of the aponeurosis of the thenar and hypothenar muscles distally, the transverse carpal ligament, and the investing fascia of the distal forearm proximally [8]. The typical patient experiences nocturnal parasthesias, numbness, and proximal radiation of pain to the forearm and arm. If the condition worsens, numbness in the median nerve distribution, together with weakness of the thenar muscles, can occur [1]. The open carpal 
tunnel release (OCTR) is considered till now the standard procedure as it is easy, rapid, of minimal cost, sure under-vision complete release of the tunnel and has provided good long-term results, but has been associated with pillar pain, slow return of pinch strength, and slow return to work with minimal possibility of transient neurapraxia $(0.25 \%)$. On the other hand, several studies have supported that the endoscopic carpal tunnel release (ECTR) patients have less postoperative pain, faster return to functional activities, and early improvement of hand grip and pinch strength but with higher possibility of transient neurapraxia $(1.45 \%)$. After 1 year, the difference in early results between both open and endoscopic techniques disappears and the late results were found the same [9]. The endoscopic carpal tunnel release (ECTR) is not a popular technique used for treatment for this disease in Egypt due to higher cost, longer time and fear of incomplete release of the carpal ligament and transient median neuropathy. So, it was needed to perform a study to evaluate the endoscopic technique for carpal tunnel release as regard safety, efficacy and if it adds more benefits to our patients.

\section{Patients and Methods}

The present study is a prospective descriptive study performed over 2 years, between September 2014 and September 2016 on 36 hands in 36 patients admitted to Al-Azhar university hospitals, Cairo, Egypt. All patients had symptoms of idiopathiccarpal tunnel syndrome as pain, numbness, paresthesias or weakness in the distribution of the median nerve in one or both hands with proximal radiation of the pain to forearm or arm. In cases with bilateral complain, one hand was more severe. Clinical signs included tenderness and muscle atrophy. Thenar bulk and contour were assessed where atrophy was recorded as 0 (absent), 1 (mild), 2 (moderate), or 3 (severe). The strength of the abductor pollicis brevis was measured and graded from 0 to 5 and positive provocative tests as Tinel and Phalen tests and electro-diagnostic findings consistent with moderate to severe carpal tunnel syndrome and had not responded to non-operative management. Electrophysiological studies showed distal motor latency values greater than 4.5 milliseconds or there was a difference in values between the affected and the healthy hands of one millisecond or more. The sensory latency was more than 3.5 milliseconds and/or 0.5 milli-second more than that on the opposite side. All patients had not responded to primary non-operative management for at least 3 months. Conservative treatment included non-steroidal anti-inflammatory drugs, gabapentin, pregabaline, vitamin B complex, steroids for some patients, hand rest, hand splinting and physiotherapy.

Patients included in this study were all patients with confirmed diagnosis of unilateral or bilateral carpal tunnel syndrome of any age and any sex whom considered for surgical release for first time after failure of 3 month conservative treatment. Patients excluded from the study were those with recurrent carpal tunnel syndrome after open or endoscopic techniques, diabetes mellitus and 
those secondary to rheumatologic diseases or acromegaly, patients with limited wrist extension, associated Dupuytren's contracture, and masses in the carpal canal.

\section{Ethical Considerations}

Written informed consents were obtained from all participant patients after full explanation of the surgical technique and that their demographic and clinical data will be included in the study while patient identities will not be included. This study was approved by the ethics committee of our department.

Surgical technique used [10]: under local anesthesia, single portal 1 centimeter transverse incision between the flexor carpi ulnaris and palmaris longus tendons was performed at or just proximal to the proximal wrist crease. Subcutaneous fat was separated till the transverse carpal ligament was visualized. A 5 millimeter incision was made in the transverse carpal ligament longitudinally in a U-shaped fashion. Blunt dissection was used to clear the synovial membrane from the undersurface of the ligament. With the wrist placed in 30 degrees of extension. The smaller then the larger dilators were wet with sterile saline then passed gentlybelow the ligament along the tunnel for many times to well develop space between the median nerve and the transverse carpal ligament steering in the direction of the ring finger axis without producing excessive compression of the already compressed median nerve in the narrow tunnel until the tip of the dilators was palpated in the palm. The trocar and sheath assembly were then inserted also till the tip was palpated in the palm. The trocar was then removed and 30 degree scope was inserted through the sheath and the undersurface of the ligament was explored. A special endoscopic knife was then inserted through the slit of the sheath above the scope and the transverse carpal ligament was cut from proximal to distal gradually step by step to avoid the motor branch of the median nerve. Satisfactory release of the ligament was confirmed when subcutaneous fat droops down then the sheath was removed after replacement of the trocar. After adequate hemostasis had been obtained, small tube drain was inserted and the incision was sutured with 3 - 0 vicryl subcuticularly then a sterile dressing was applied for 24 hours (Figure 1).

Complications as injury to the median nerve or its motor or superficial palmar branches, arteries or tendons were in mind. Follow up after one week then after 1, 3 and 6 months. Patients were allowed to use the hand in the following day and return to their activities as tolerated and the time was monitored. They were advised to avoid heavy work and excessive activity associated with compression of the hand. Demographic, preoperative and postoperative data were collected.

Analysis of data for this descriptive study was expressed as frequency-percentage and mean \pm SD.

The patients were examined during the follow up period as regard grip and pinch function, palmar tenderness, and painful scar formation using the simple questions used by Aydin et al., (2006) selected from the daily activities in an 


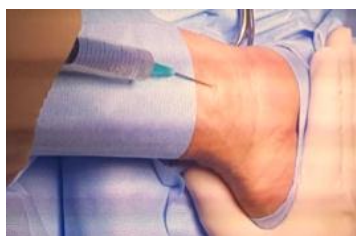

(a)

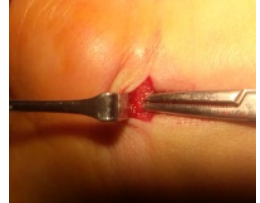

(d)

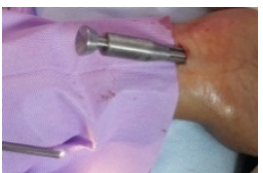

(h)

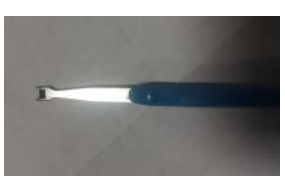

(1)

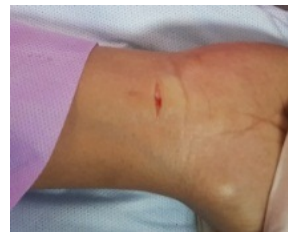

(b)

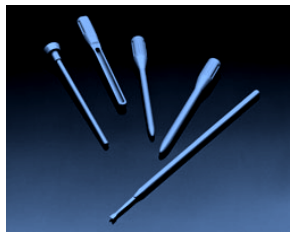

(c)

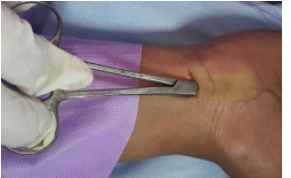

(e)

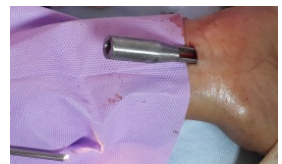

(i)

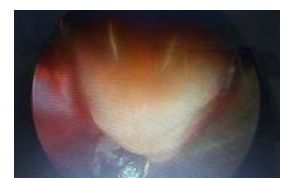

(m)

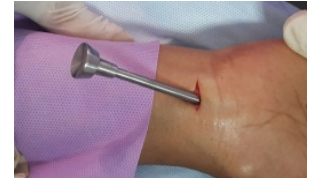

(f)

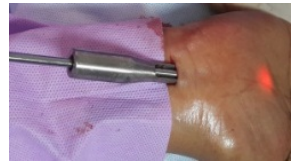

(j)

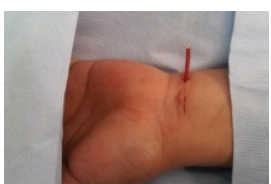

(n)

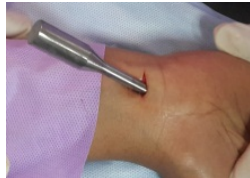

(g)

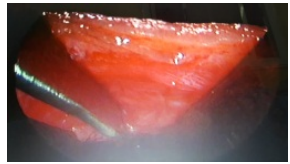

(k)

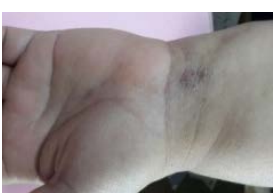

(o)

Figure 1. Steps of the surgical technique. (a) Injecting Local anesthesia; (b) Skin incision; (c) ECTR set; (d) Identifying the ligament; (e) Fat separation; (f) Passing Small dilator; (g) Passing Bigger dilator; (h) Passing trocar \& sheath; (i) Removing trocar; (j) Passing the scope; (k) Viewing the ligament; (l) Special endoscopic knife; (m) view after release; (n) Drain \&closure; (o) wound after 2 weeks.

ascending order [2]. For gripping (stick of an electrical vacuum, handle of a knife, handle of a door then stalk of a hair-drying machine) and for pinching (key turning it to open a door, pen and writing, toothbrush and brushing teeth then screwdriver).

The outcome was decided after 6 weeks of follow up and graded as poor, moderate, good, and excellent according to satisfaction of patients based on absence or presence of complications, disappearance of pain and return to work (Table 1).

\section{Results}

This is a prospective descriptive study performed over 2 years, between September 2014 and September 2016 on 36 patients admitted to Al-Azhar university hospitals, Cairo, Egypt. All patients were suffering from carpal tunnel syndrome unilaterally or bilaterally with one side was severe than the other. The duration of symptoms ranged from 6 to 18 months (mean, 8.5 months). Endoscopiccarpal tunnel release (ECTR) was the procedure that performed for 36 hands in the 36 patients. The 36 patients included 33 women (92\%) and 3 men (8\%) with marked female predominance ( $\mathrm{F}: \mathrm{M}=9: 1$ ). Their ages ranged from 26 to 58 years 
Table 1. Showing the outcome determinants.

\begin{tabular}{cccc}
\hline Outcome after 6 months & Complications & Pain & Return to work \\
\hline Excellent & No & No & Full activities \\
Good & No & No & Limited activities \\
Moderate & No & Still & Limited activities \\
Poor & complicated & still & No activities \\
\hline
\end{tabular}

with the mean age was 42 years. Among the 36 patients, 32 are of right hand dominance (90\%) and 4 are left of hand dominance (10\%).The right hand was affected in 24 patients (67\%), all are right handed and the left hand was affected in 12 patients (33\%), of them 4 are left handed (11\%) and 8 are right handed (22\%). The right to left affection ratio was 2:1. The syndrome was bilateral in 21 patients $(60 \%)$ with severe symptoms in one side and mild symptoms in the contralateral side. Of them the 12 patients with left hand affection where mild symptoms were present in the right hand and 9 patients with right hand affection and mild symptoms in left hand. In 15 patients the condition was unilateral on right side with no symptoms on left side. Preoperative symptoms included pain, numbness and weakness. Pain was found in all cases (100\%) with exaggerating on effort and after periods of rest especially in the early morning and in 12 patients (33\%) pain used to awaken them from sleep with radiation to the forearm or arm in 18 cases (50\%). Numbness and paresthesias along distribution of median nerve in the palm was found in 21 cases $(60 \%)$. Weakness of hand grip was found in 6 cases (17\%). An obvious wasting of the thenar muscle was noticed in 9 out of 36 cases (25\%) while 27 cases (75\%) had no obvious wasting. Tenderness along the tunnel found in 7 cases (20\%). Tinel's sign was positive in 10 patients (28\%) and Phalen's test was positive in 12 patients (33\%). Nerve conduction study of the median nerve showed moderate motor latency in 22 cases $(61 \%)$ and severe in 14 cases $(39 \%)$ while sensory latency was moderate in 27 cases (75\%) and severe in 9 cases $(25 \%)$.

Conservative treatment was given to all patients for at least 3 months with no response where endoscopic release was performed for all patients after informed consent. The average operating time was 10 minutes (range $8-15 \mathrm{mi}-$ nutes).

There were no intraoperative complications as bleeding or injury to nerves (median nerve, motor branch of median nerve or palmar cutaneous branch of median nerve), tendons, or arteries in all patients. In 3 patients (8\%), transient neurapraxia in the long/ring web space occurred and resolved in 1 week with the aid of anti-inflammatory and neurotonic drugs.

The mean follow up period was 6 months. There were no cases of postoperative infection or recurrence within the study period. Out of 36 cases, post operative tenderness of the skin over the tunnel was found in 26 cases (70\%) at 1 week and in 10 cases (28\%) at 2 weeks while in 1 case (3\%) at 4 weeks. After 6 weeks, all patients showed major or complete relief of symptoms. Postoperative, Tinel 
sign and Phalen test were positive in 8 and 10 cases respectively and both became negative in 1 month. There was an initial reduction in hand grip and pinch strength at 2 weeks, followed by a significant improvement in 1 month onwards.

The outcome after 6 months was: excellent in 25 cases (70\%), good in 10 cases (27\%), moderate in 1 case (3\%) and no cases of poor outcome.

\section{Discussion}

Because of less surgical trauma, less postoperative pain, less scar formation, better cosmetic result and short recovery time, endoscopic release of idiopathic carpal tunnel syndrome was highly demanded especially with development of good endoscopic systems and rising experience with endoscopic carpal tunnel surgery. It was reported that open carpal tunnel release is associated with considerable morbidity, including prolonged tenderness of the scar and weakness of grip for as long as six months after the operation [3]. Reports of the results of a double-blind multicenter prospective randomized clinical study of the one portal carpal tunnel release [5]. These results indicate that there is less morbidity with a closed endoscopic carpal tunnel release than with an open operation. The possibility of improvement of these outcomes has generated considerable interest in the endoscopic method [5]. Many studies reported that open carpal tunnel release procedure provides lasting alleviation of symptoms in more than $80 \%$ of patients and the endoscopic method provides relief of numbness and paresthesias in higher percentage [2] [11].

A study with results of endoscopic carpal-tunnel release noted that $59 \%$ of patients returned to normal activities and to work after 2 weeks and $86 \%$, after 4 weeks [4].

\section{Limitations}

This study has many limitations. First, the cost of endoscopic technique is double that of open one. Second, the number of patients is small, so, the real outcome of this technique in particular needs further studies with greater number of patients and following different techniques in comparison form.

\section{Conclusion}

Endoscopic carpal tunnel release is an effective safe minimal invasive procedure with high success rate, rapid return to work and minimal early and late complications.

\section{Acknowledgements}

For all my colleagues of department of neurosurgery, Al-Azhar University.

\section{Conflicts of Interest}

There are no conflicts of interest. 


\section{References}

[1] Aydin, K., Cokluk, C., Cengiz, N. and Bilgici, A. (2006) Microsurgical Open Mini Uniskin Incision Technique in the Surgical Treatment of Carpal Tunnel Syndrome. Neurology India, 54.

[2] Van Heest, A., Waters, P., Simmons, B. and Schwartz, J. (1995) A Cadaveric Study of the Single-Portal Endoscopic Carpal Tunnel Release. Journal of Hand Surgery, 20A, 363-366. https://doi.org/10.1016/S0363-5023(05)80088-7

[3] Ravindran, T., Sara, T. and Loh, Y. (2009) Endoscopic Carpal Tunnel Release-A Prospective Study of Functional Outcome. Malaysian Orthopaedic Journal, 3. https://doi.org/10.5704/MOJ.0905.008

[4] Chow, J. (1989) Endoscopic Release of the Carpal Ligament: A New Technique for Carpal Tunnel Syndrome. Arthroscopy, 5, 19-24. https://doi.org/10.1016/0749-8063(89)90085-6

[5] Agee, J., Mc Carroll, H., Tortosa, R., Berry, D., Szabo, R. and Peimer, C. (1992) Endoscopic; Release of the Carpal Tunnel: A Randomized Prospective Multicenter Study. Journal of Hand Surgery, 17A, 987-995. https://doi.org/10.1016/S0363-5023(09)91044-9

[6] Okutsu, I. (1989) Endoscopic Management of Carpal Tunnel Syndrome. Arthroscopy, 5, 11-18. https://doi.org/10.1016/0749-8063(89)90084-4

[7] Bartłomiej, H., Noszczy, K., Natalia, K. and Marcin, N. (2014) Superficial Plane Endoscopy for Carpal Tunnel Release Video Surgery. Mini Inv, 9, 262-266.

[8] Cobb, T., Dally, B. and Posteraro, R. (1993) Anatomy of the Flexor Retinaculum. Journal of Hand Surgery, 18A, 89-91.

[9] Chow, J., Hantes, M. and Vernon, M. (2002) Endoscopic Carpal Tunnel Release: Thirteen Years of Experience with the Chow Technique. The Journal of Hand Surgery.

[10] Tuzuner, S., Sherman, G., Ozkaynak, S. and Ozcanli, H. (2004) Endoscopic Carpal Tunnel Release: Modification of Menon's Technique and Data from 191 Cases. Arthroscopy, 20, 721-727. https://doi.org/10.1016/S0749-8063(04)00588-2

[11] Ruch, D. and Poehling, G. (1996) Endoscopic Carpal Tunnel Release: Agee Technique. Hand Clinics, 12, 299-303. 